\title{
Analytical Analysis on Nonlinear Parametric Vibration of an Axially Moving String with Fractional Viscoelastic Damping
}

\author{
Ying $\mathrm{Li}^{1}$ and Ye Tang ${ }^{2}$ \\ ${ }^{1}$ School of Arts, Anhui Polytechnic University, Wuhu 241000, China \\ ${ }^{2}$ School of Mechanical and Automotive Engineering, Anhui Polytechnic University, Wuhu 241000, China \\ Correspondence should be addressed to Ye Tang; tangye2010_hit@163.com
}

Received 14 May 2017; Accepted 25 October 2017; Published 16 November 2017

Academic Editor: Jaromir Horacek

Copyright (c) 2017 Ying Li and Ye Tang. This is an open access article distributed under the Creative Commons Attribution License, which permits unrestricted use, distribution, and reproduction in any medium, provided the original work is properly cited.

\begin{abstract}
The nonlinear parametric vibration of an axially moving string made by rubber-like materials is studied in the paper. The fractional viscoelastic model is used to describe the damping of the string. Then, a new nonlinear fractional mathematical model governing transverse motion of the string is derived based on Newton's second law, the Euler beam theory, and the Lagrangian strain. Taking into consideration the fractional calculus law of Riemann-Liouville form, the principal parametric resonance is analytically investigated via applying the direct multiscale method. Numerical results are presented to show the influences of the fractional order, the stiffness constant, the viscosity coefficient, and the axial-speed fluctuation amplitude on steady-state responses. It is noticeable that the amplitudes and existing intervals of steady-state responses predicted by Kirchhoff's fractional material model are much larger than those predicted by Mote's fractional material model.
\end{abstract}

\section{Introduction}

Axially moving structures are one of common elements in many mechanical systems, which is extensively used in engineering fields, such as paper sheets, magnetic tapes, power transmission belts, chains, fiber textiles, aerial tramways, pipes conveying fluids, and thread lines. In many cases, due to initial, parametric, and external excitations, the generation of unwanted transverse vibrations may limit their applications. Therefore, the dynamic behaviors of such devices have been widely investigated by numerous scholars for the past decades and are still of interesting today [1-10]. Moreover, according to the demand of the actual engineering problems, some researchers have applied their theoretical results to design and optimize the axially moving structures [11, 12].

In general, small imperfections induced by either geometrical or dynamic sources may bring about the occurrence of an unsteady axial speed or tension. For example, when an axially moving belt is installed on rotating pulleys, the torsional vibration of the pulleys would lead to a small fluctuation in the axial moving velocity, and consequently this system may exhibit more complicated dynamics behaviors like the parametric resonances. Thus, the parametric resonances of the axially moving structures caused by the pulsatile transport speed or tension have received extensive attention. Fung et al. [13] applied the Galerkin method and the numerical integration technique to investigate the parametric vibration of a viscoelastic string with the nonuniform transport speed. Pellicano et al. [14] used the approximate analytical and the experimental methods to study the primary and the parametric resonances of a power transmission belt with the fluctuation of the tension. Chen et al. [15] employed the averaging method to investigate the stability problems of an axially accelerating tensioned beam under the condition of the subharmonic and combination resonances. Pakdemirli and Öz [16] adopted a perturbation technique to discuss stable regions of a simply supported axially moving beams subjected to sum- and difference-type combination resonances. Ghayesh [17] utilized the method of multiple scales to study the stability characteristics for principal and combination parametric resonances of an axially moving string with the partial elastic support. 
With the development of engineering technique, the various complicated materials are utilized to fabricate the axially moving structures. In order to better understand energy dissipation mechanism of such materials, some viscoelastic constitutive relations like the Kelvin-Voigt form have been used to describe mechanical behaviors of the materials, which is widely applied in axially moving continuums such as strings $[18,19]$, beams $[20,21]$, belts $[22,23]$, and plates $[24$, $25]$. In addition, some structures made of more complex viscoelastic materials are explored. Marynowski and Kapitaniak [26] presented a mathematical model of an axially moving viscoelastic beam with the three-parameter Zener element and investigated both regular and chaos motions using the Galerkin method. Chen et al. [27] analytically studied nonlinear parametric responses of an axially moving string composed of the complicated viscoelastic material based on the Boltzmann superposition principle. Wang and Chen [28] developed the differential quadrature scheme to determine the stable boundary of an axially moving viscoelastic beam with the standard linear solid in the case of the principal resonance. Ding and Chen [29] employed the material time derivative to characterize the viscoelastic property of an axially moving viscoelastic beam. They investigated the stability in principle resonance of the system by using analytical and numerical methods.

On the other hand, the axially moving structures made by rubber-like materials are widely applied in some engineering fields. To exactly describe the viscoelastic features, some scholars have adopted the fractional derivative theory to model the structures. For example, Chen et al. [30] established the fractional dynamic model of an axially moving string and analyzed the transient responses using the Galerkin and numerical methods. Yang et al. [31, 32] employed the multiscale method to investigate the nonlinear free and parametric vibrations of an axially moving viscoelastic string with a fractional order damping.

The above-mentioned studies are concentrated on axially moving strings based on Mote's model. Besides, the nonlinear integro-partial-differential equation called Kirchhoff's model is also used for describing the transverse motion of axially moving strings [33-35]. Nevertheless, the applications of Kirchhoff's model in axially moving structures obeying the fractional differentiation law are rather limited, and the difference of fractional parametric resonances between the Mote's model and the Kirchhoff's model remains unclear. Therefore, the present paper further explores the nonlinear parametric vibration of an axially moving string with the fractional viscoelastic damping based on the literature [32].

\section{Equations of Motion}

Consider a uniform, axially moving viscoelastic string made by rubber-like materials, with linear density $\rho$, the length $L$, and the length cross-sectional area $A$, traveling at timedependent axially speed $V(\widehat{t})$ between two fixed supports at both ends, shown in Figure 1 . The symbol $\widehat{u}(\widehat{x}, \widehat{t})$ represents the transverse displacement, $\widehat{x}$ is the coordinate along the axial direction, and $\hat{t}$ is the time. On the basis of the Euler beam theory and the Newton second law [34], the Kirchhoff's mathematical model governing transverse motion can be obtained as

$$
\begin{gathered}
\rho A\left(\frac{\partial^{2} \widehat{u}}{\partial \widehat{t}^{2}}+2 V \frac{\partial^{2} \widehat{u}}{\partial \widehat{x} \partial \widehat{t}}+\frac{d V}{d \widehat{t}} \frac{\partial \widehat{u}}{\partial \widehat{x}}+V^{2} \frac{\partial^{2} \widehat{u}}{\partial \widehat{x}^{2}}\right)-P_{0} \frac{\partial^{2} \widehat{u}}{\partial \widehat{x}^{2}} \\
-\frac{\partial^{2} \widehat{u}}{\partial \widehat{x}^{2}} \frac{1}{L} \int_{0}^{L} A \widehat{\sigma}(\widehat{x}, \hat{t}) d \widehat{x}=0,
\end{gathered}
$$

where $P_{0}$ represents the initial tension and $\widehat{\sigma}(\widehat{x}, \widehat{t})$ denotes the axial disturbed stress. According to the Lagrangian strain [35], the disturbed strain accounted for the geometric nonlinearity can be expressed as

$$
\varepsilon_{L}(\widehat{x}, \widehat{t})=\frac{1}{2}\left(\frac{\partial \widehat{u}}{\partial \widehat{x}}\right)^{2}
$$

In the work, the viscoelastic damping of the string is supposed as obeying the fractional derivative Kelvin-Voigt model [32]. Thus, the stress-strain relation can be given as

$$
\widehat{\sigma}(\hat{x}, \widehat{t})=E_{0} \varepsilon_{L}(\widehat{x}, \widehat{t})+E_{1} \frac{\partial^{\alpha}}{\partial \widehat{t}^{\alpha}}{ }_{R L+} \varepsilon_{L}(\widehat{x}, \widehat{t}),
$$

where $E_{0}$ and $E_{1}$ represent the stiffness constant and viscosity coefficient of the string, respectively, and $\alpha$ is a constant which is used to describe the viscosity characteristic. Considering the fractional derivative operator defined by the RiemannLiouville form $[36,37]$, we write the expression $\partial^{\alpha} / \partial \widehat{t}^{\alpha}{ }_{R L+}(\mathrm{l})$ in (3) as follows:

$$
{\frac{\partial^{\alpha}}{\partial \widehat{t}^{\alpha}}}_{R L+} \varepsilon_{L}=\frac{1}{\Gamma(1-\alpha)} \frac{d}{d \widehat{t}} \int_{0}^{\widehat{t}}(\widehat{t}-\tau)^{-\alpha} \varepsilon_{L}(\tau) d \tau,
$$

where $\Gamma$ is the Gamma function. The fractional derivative operator is applied to investigate an intermediate viscoelastic characteristic between the elastic string $(\alpha=0)$ and KelvinVoigt viscoelastic string $(\alpha=1)$. For calculating the fractional differentiation operator, we introduce the following property as

$$
\frac{d^{\alpha}}{d \widehat{t}^{\alpha}}{ }_{R L+}^{i \omega \hat{t}}=(i \omega)^{\alpha} e^{i \omega \hat{t}}+\frac{\sin (\pi \alpha)}{\pi} \int_{0}^{\infty} \frac{u^{\alpha}}{u+i \omega} e^{-u \widehat{t}} d u
$$

where $i$ denotes complex number $\sqrt{-1}$.

Substituting (2), (3), and (4) into (1), the mathematical model of transverse motion for the axially moving viscoelastic string can be obtained as

$$
\begin{gathered}
\rho A\left(\frac{\partial^{2} \widehat{u}}{\partial \widehat{t}^{2}}+2 V \frac{\partial^{2} \widehat{u}}{\partial \widehat{x} \partial \widehat{t}}+\frac{d V}{d T} \frac{\partial \widehat{u}}{\partial \widehat{x}}+V^{2} \frac{\partial^{2} \widehat{u}}{\partial \widehat{x}^{2}}\right)-P_{0} \frac{\partial^{2} \widehat{u}}{\partial \widehat{x}^{2}} \\
=\frac{E_{0} A}{2 L} \frac{\partial^{2} \widehat{u}}{\partial \widehat{x}^{2}} \int_{0}^{L}\left(\frac{\partial \widehat{u}}{\partial \widehat{x}}\right)^{2} d \widehat{x} \\
+\frac{E_{1} A}{2 L} \frac{\partial^{2} \widehat{u}}{\partial \widehat{x}^{2}} \int_{0}^{L} \frac{\partial^{\alpha}}{\partial t^{\alpha}}\left(\frac{\partial \widehat{u}}{\partial \widehat{x}}\right)^{2} d \widehat{x} .
\end{gathered}
$$

For the axially moving string with two fixed supports, the boundary conditions are satisfied by

$$
u(0, \widehat{t})=u(L, \widehat{t})=0
$$




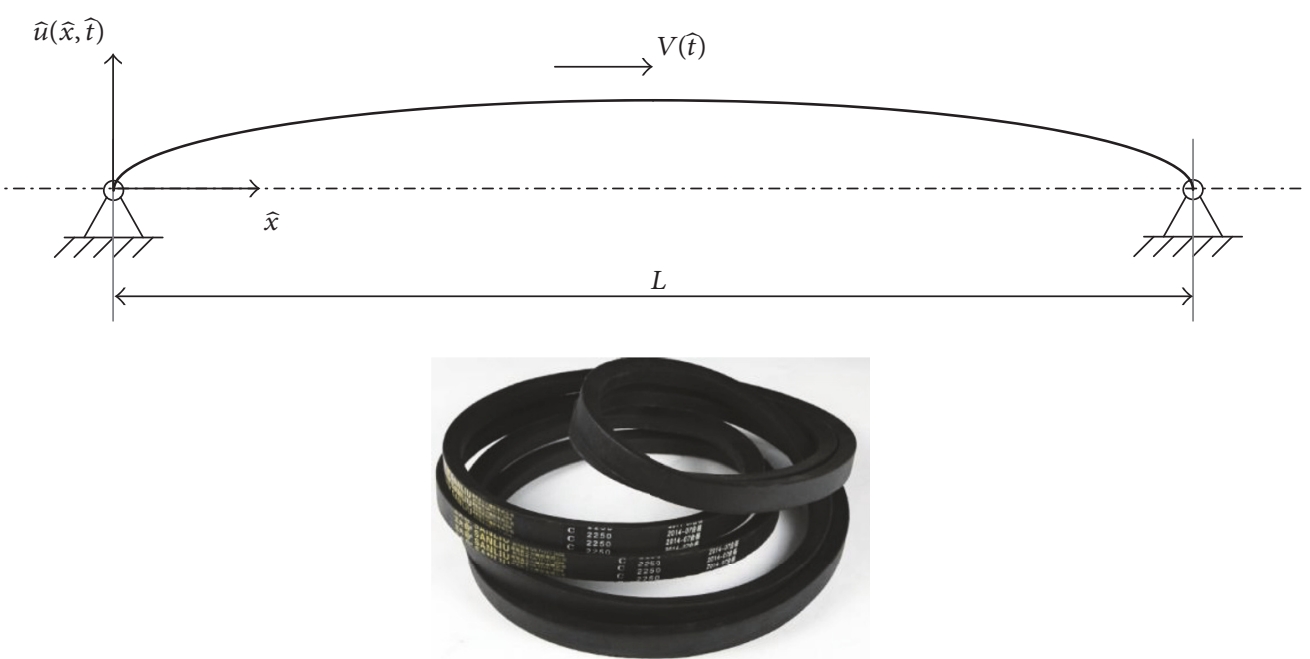

FIGURE 1: The physical model of the axially moving strings made by rubber-like materials.

Introduce the dimensionless variables

$$
\begin{aligned}
u & =\frac{\widehat{u}}{L}, \\
x & =\frac{\widehat{x}}{L}, \\
t & =\frac{\widehat{t}}{L} \sqrt{\frac{P_{0}}{\rho A}}, \\
\gamma & =V \sqrt{\frac{\rho A}{P_{0}}}, \\
\varepsilon e_{0} & =\frac{E_{0} A}{P_{0}}, \\
\varepsilon \eta & =\frac{E_{1} A P^{\alpha / 2-1}}{\sqrt{\left(\rho A L^{2}\right)^{\alpha}}},
\end{aligned}
$$

where the book-keeping device $\varepsilon$ is a small parameter, indicating that both the stiffness constant and the viscosity coefficient are very small. Substituting (8) into (6) and (7), one can obtain the following dimensionless dynamic equation,

$$
\begin{aligned}
\frac{\partial^{2} u}{\partial t^{2}}+ & 2 \gamma \frac{\partial^{2} u}{\partial x \partial t}+\frac{d \gamma}{d t} \frac{\partial u}{\partial x}+\left(\gamma^{2}-1\right) \frac{\partial^{2} u}{\partial x^{2}} \\
= & \frac{1}{2} \varepsilon e_{0} \frac{\partial^{2} u}{\partial x^{2}} \int_{0}^{1}\left(\frac{\partial u}{\partial x}\right)^{2} d x \\
& +\frac{1}{2} \varepsilon \eta \frac{\partial^{2} u}{\partial x^{2}} \int_{0}^{1} \frac{\partial^{\alpha}}{\partial t^{\alpha}}\left(\frac{\partial u}{\partial x}\right)^{2} d x
\end{aligned}
$$

and boundary conditions,

$$
u(0, t)=u(1, t)=0 .
$$

In order to study parametric vibrations, we suppose that the axial moving speed is a small harmonically varying about the constant mean speed $\gamma_{0}$ :

$$
\gamma=\gamma_{0}+\varepsilon \gamma_{1} \sin (\Omega t)
$$

where $\gamma_{1}$ and $\Omega$ are the fluctuation amplitude and frequency, respectively. Substituting (11) into (9) yields

$$
\begin{aligned}
\frac{\partial^{2} u}{\partial t^{2}} & +2 \gamma_{0} \frac{\partial^{2} u}{\partial x \partial t}+\frac{d \gamma}{d t} \frac{\partial u}{\partial x}+\left(\gamma_{0}^{2}-1\right) \frac{\partial^{2} u}{\partial x^{2}} \\
= & \varepsilon\left[\frac{1}{2} e_{0} \frac{\partial^{2} u}{\partial x^{2}} \int_{0}^{1}\left(\frac{\partial u}{\partial x}\right)^{2} d x\right. \\
+ & \frac{1}{2} \eta \frac{\partial^{2} u}{\partial x^{2}} \int_{0}^{1} \frac{\partial^{\alpha}}{\partial t^{\alpha}}\left(\frac{\partial u}{\partial x}\right)^{2} d x-\gamma_{1} \Omega \cos (\Omega t) \frac{\partial u}{\partial x} \\
- & \left.2 \gamma_{1} \sin (\Omega t) \frac{\partial^{2} u}{\partial x \partial t}-2 \gamma_{0} \gamma_{1} \sin (\Omega t) \frac{\partial^{2} u}{\partial x^{2}}\right]
\end{aligned}
$$

\section{Analytical Treatments}

Equation (12) is a fractional, nonlinear integro-partialdifferential equation; the direct multiscale method is applied to solve this equation for finding analytical solutions. Considering a first-order uniform approximation, one gives

$$
u(x, t, \varepsilon)=u_{0}\left(x, T_{0}, T_{1}\right)+\varepsilon u_{1}\left(x, T_{0}, T_{1}\right)+\cdots,
$$

where $u_{0}$ and $u_{1}$ are the displacement functions at orders 1 and $\varepsilon, T_{0}=t$ and $T_{1}=\varepsilon t$ are the fast and slow time scales, respectively. Substituting (13) into (12) and then equalizing the coefficients $\varepsilon^{0}$ and $\varepsilon$ lead to

$$
\begin{aligned}
& \varepsilon^{0}: \frac{\partial^{2} u_{0}}{\partial T_{0}^{2}}+2 \gamma_{0} \frac{\partial^{2} u_{0}}{\partial T_{0} \partial x}+\left(\gamma_{0}^{2}-1\right) \frac{\partial^{2} u_{0}}{\partial x^{2}}=0 \\
& \varepsilon^{1}: \frac{\partial^{2} u_{1}}{\partial T_{0}^{2}}+2 \gamma_{0} \frac{\partial^{2} u_{1}}{\partial T_{0} \partial x}+\left(\gamma_{0}^{2}-1\right) \frac{\partial^{2} u_{1}}{\partial x^{2}}
\end{aligned}
$$




$$
\begin{aligned}
= & -2 \frac{\partial^{2} u_{0}}{\partial T_{0} \partial T_{1}}-2 \gamma_{0} \frac{\partial^{2} u_{0}}{\partial x \partial T_{1}} \\
& +\frac{1}{2} e_{0} \frac{\partial^{2} u_{0}}{\partial x^{2}} \int_{0}^{1}\left(\frac{\partial u}{\partial x}\right)^{2} d x \\
& +\frac{1}{2} \eta \frac{\partial^{2} u_{0}}{\partial x^{2}} \int_{0}^{1}\left(\frac{\partial}{\partial T_{0}}\right)_{R L+}^{\alpha}\left(\frac{\partial u}{\partial x}\right)^{2} d x \\
& -\gamma_{1} \Omega \cos \left(\Omega T_{0}\right) \frac{\partial u_{0}}{\partial x}-2 \gamma_{1} \sin \left(\Omega T_{0}\right) \frac{\partial^{2} u_{0}}{\partial x \partial T_{0}} \\
& -2 \gamma_{0} \gamma_{1} \sin \left(\Omega T_{0}\right) \frac{\partial^{2} u_{0}}{\partial x^{2}} .
\end{aligned}
$$

At $\varepsilon^{0}$, the solution can be written as follows:

$$
\begin{aligned}
u_{0}\left(x, T_{0}, T_{1}\right)= & A_{n}\left(T_{1}\right) \phi_{n}(x) e^{i \omega_{n} T_{0}} \\
& +\bar{A}_{n}\left(T_{1}\right) \bar{\phi}_{n}(x) e^{-i \omega_{n} T_{0}},
\end{aligned}
$$

where $A_{n}$ is a complex function which is only related to the slow time scale $T_{1}$, the overbar represents the complex conjugate, and $\omega_{n}$ and $\phi_{n}(x)$ are the $n$th natural frequency and mode function of the corresponding linear system, which is given by the following [1]:

$$
\begin{aligned}
\omega_{n} & =n \pi\left(1-\gamma_{0}^{2}\right), \\
\phi_{n}(x) & =\sqrt{2} \sin (n \pi x) e^{i n \pi \gamma_{0} x} .
\end{aligned}
$$

It is widely known that the principal parametric resonance may occur if $\Omega$ approaches $2 \omega_{n}$. Thus, the nearness relation can be supposed as follows:

$$
\Omega=2 \omega_{n}+\varepsilon \sigma,
$$

in which $\sigma$ is a detuning parameter.

Substituting (16), (17), and (18) into (15), one yields the following solvability condition $[38,39]$ :

$$
\frac{d A_{n}}{d T_{1}}+\chi_{1} A_{n}{ }^{2} \bar{A}_{n}+\chi_{2} \bar{A}_{n} e^{i \sigma T_{1}}=0
$$

where

$$
\begin{aligned}
& \chi_{1}=\frac{-2 e_{0} \int_{0}^{1} \bar{\phi}_{n}^{\prime \prime} \bar{\phi}_{n} d x \int_{0}^{1} \phi_{n}^{\prime} \bar{\phi}_{n}^{\prime} d x+\left[e_{0}+\eta\left(2 i \omega_{n}\right)^{\alpha}\right] \int_{0}^{1} \bar{\phi}_{n}^{\prime \prime} \bar{\phi}_{n} d x \int_{0}^{1} \phi_{n}^{\prime 2} d x}{4\left[i \omega_{n} \int_{0}^{1} \phi_{n} \bar{\phi}_{n} d x+\gamma_{0} \int_{0}^{1} \phi_{n}^{\prime} \bar{\phi}_{n} d x\right]}, \\
& \chi_{2}=-\frac{\left(\omega_{n}-\Omega / 2\right) \int_{0}^{1} \bar{\phi}_{n}^{\prime} \bar{\phi}_{n} d x+2 \gamma_{0} \int_{0}^{1} \bar{\phi}_{n}^{\prime \prime} \bar{\phi}_{n} d x}{2\left[i \omega_{n} \int_{0}^{1} \phi_{n} \bar{\phi}_{n} d x+\gamma_{0} \int_{0}^{1} \phi_{n}^{\prime} \bar{\phi}_{n} d x\right]} \gamma_{1},
\end{aligned}
$$

where $\chi_{1}$ and $\chi_{2}$ are the constant coefficients depending on the fraction order, viscosity, stiffness constant, axial moving speed, and the linear frequency. Separating these coefficients into real and image parts, one yields

$$
\begin{aligned}
& \chi_{1}=\operatorname{Re}\left(\chi_{1}\right)+i \operatorname{Im}\left(\chi_{1}\right) \\
& \chi_{2}=\operatorname{Re}\left(\chi_{2}\right)+i \operatorname{Im}\left(\chi_{2}\right) .
\end{aligned}
$$

Express the solution to (19) in the polar form:

$$
A_{n}=\frac{1}{2} a_{n}\left(T_{1}\right) e^{i \phi_{n}\left(T_{1}\right)}
$$

in which real functions $a_{n}$ and $\phi_{n}$ are the amplitude and phase of the $n$th resonance, respectively. Substituting of (21) and (22) in (19) and then separating real and imaginary parts, we have

$$
\begin{aligned}
\frac{d a_{n}}{d T_{1}}= & a_{n}\left[\operatorname{Im}\left(\chi_{2}\right) \sin \theta_{n}-\operatorname{Re}\left(\chi_{2}\right) \cos \theta_{n}\right] \\
& -\frac{1}{4} \operatorname{Re}\left(\chi_{1}\right) a_{n}^{3}
\end{aligned}
$$

$$
\begin{aligned}
\frac{d \theta_{n}}{d T_{1}}= & \sigma+2\left[\operatorname{Re}\left(\chi_{2}\right) \sin \theta_{n}-\operatorname{Im}\left(\chi_{2}\right) \cos \theta_{n}\right] \\
& +\frac{1}{2} \operatorname{Im}\left(\chi_{1}\right) a_{n}{ }^{2}
\end{aligned}
$$

where

$$
\theta_{n}=\sigma T_{1}-2 \phi_{n}
$$

For steady-state responses, the amplitude $a_{n}$ and the new phase $\theta_{n}$ should be constants; thus,

$$
\begin{aligned}
a_{n} & {\left[\operatorname{Im}\left(\chi_{2}\right) \sin \theta_{n}-\operatorname{Re}\left(\chi_{2}\right) \cos \theta_{n}\right] } \\
& -\frac{1}{4} \operatorname{Re}\left(\chi_{1}\right) a_{n}{ }^{3}=0, \\
\sigma+ & 2\left[\operatorname{Re}\left(\chi_{2}\right) \sin \theta_{n}-\operatorname{Im}\left(\chi_{2}\right) \cos \theta_{n}\right] \\
+ & \frac{1}{2} \operatorname{Im}\left(\chi_{1}\right) a_{n}{ }^{2}=0 .
\end{aligned}
$$




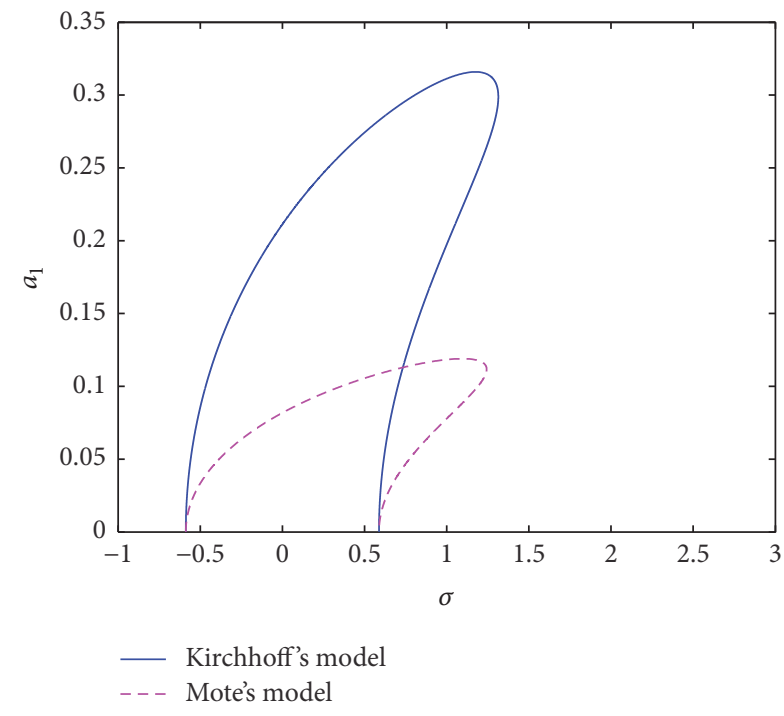

(a) The first-order mode

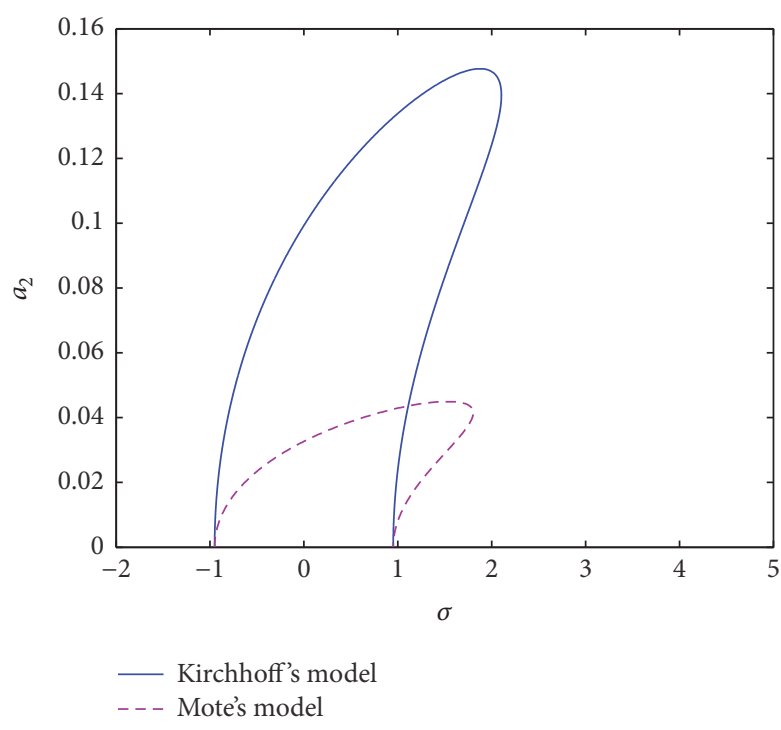

(b) The second-order mode

FIgURE 2: Comparison the steady-state responses between Kirchhoff's and Mote's fractional model.

Eliminating phase $\theta_{n}$ from (25), we can yield the amplitude of steady-state responses:

$$
a_{n}=\frac{\sqrt{-2 \sigma \operatorname{Im}\left(\chi_{1}\right) \pm 2 \sqrt{4 \chi_{1}^{2} \chi_{2}^{2}-\sigma^{2}\left[\operatorname{Re}\left(\chi_{1}\right)\right]^{2}}}}{\left|\chi_{1}\right|},
$$

where the positive and the minus signs represent the bigger and the smaller amplitudes, respectively. From [27, 28], it is proved that the bigger (smaller) response amplitude is stable (unstable). In addition, (26) is the analytical expression for steady-state responses of the string in principle parametric resonances, where the existing condition of the steady-state responses should be satisfied by

$$
\mp 2\left|\chi_{1}\right| \leq \sigma \leq \frac{2\left|\chi_{1}\right|\left|\chi_{2}\right|}{\operatorname{Re}\left(\chi_{2}\right)} .
$$

\section{Numerical Results and Discussion}

In the section, based on (26) and (27), the numerical results are presented to discuss the influences of physical parameters like the fractional order on the amplitudes and the existing conditions of steady-state responses in the principle parametric resonances of the first two modes.

Here we choose the parameters as $\gamma_{0}=0.2, \gamma_{1}=0.5$, $e_{0}=0.5, \eta=1$, and $\alpha=0.5$; the differences of steady-state responses for the string based on Kirchhoff's and Mote's fractional model are shown in Figures 2(a) and 2(b). It can be seen that the response amplitudes and the upper boundaries of exiting conditions predicted by Kirchhoff's model are much larger than those predicted by Mote's model. This implies that the application of the Kirchhoff's fractional model in axially moving strings could yield greater security prediction in the engineering. Therefore, we study the parametric resonances of the fractional damping string based on Kirchhoff's model in the next section.
The influence of the fractional order on steady-state responses for Kirchhoff's model is illustrated in Figures 3(a) and $3(\mathrm{~b})$. It is revealed that the response amplitudes decrease with the fractional order increases. Moreover, decreasing the fractional order leads to a larger upper boundary of the existing conditions, but the lower boundary is independent of the fractional order. It is indicated that the larger fractional order would lead to the more evident influence of the damping and the decreasing in existing intervals of steady-state responses. Besides, the response amplitude in the first parametric resonance is much larger than that in the second one.

The changes of steady-state responses with the stiffness constant and the viscosity coefficient of the string for the Kirchhoff's fractional model are plotted in Figures 4 and 5 , respectively. It can be demonstrated that the response amplitudes decrease dramatically with the increasing of the stiffness constant and the viscosity coefficient. Moreover, the larger the stiffness constant, the larger the existing intervals of steady-state responses. However, a smaller viscosity coefficient can increase the existing intervals, which is in accordance with in the previous viscoelastic string.

Figure 6 depicts the effect of the axial-speed fluctuation amplitude on steady-state responses for Kirchhoff's fractional string. It is observed that an increase in the axial-speed fluctuation amplitude causes the higher in both the response amplitudes and the existing intervals of steady-state responses. Therefore, we can conclude that the fractional damping string with a smaller axial-speed fluctuation amplitude has a smaller steady-state response.

\section{Conclusions}

In this study, on the basis of the fractional definition and calculus law of the Riemann-Liouville form, the nonlinear parametric vibration of an axially moving string with the fractional damping is investigated based on Kirchhoff's 


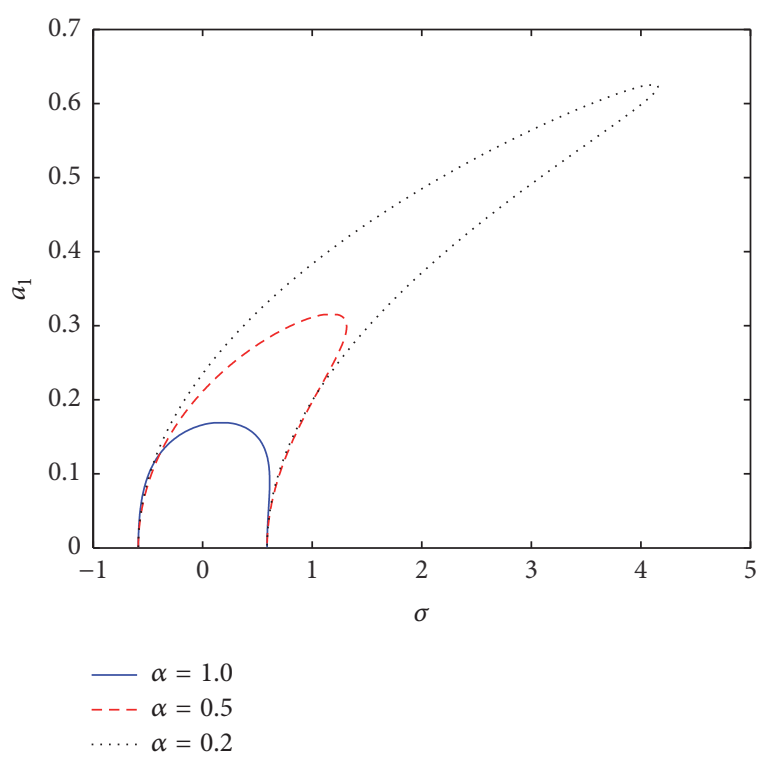

(a) The first-order mode

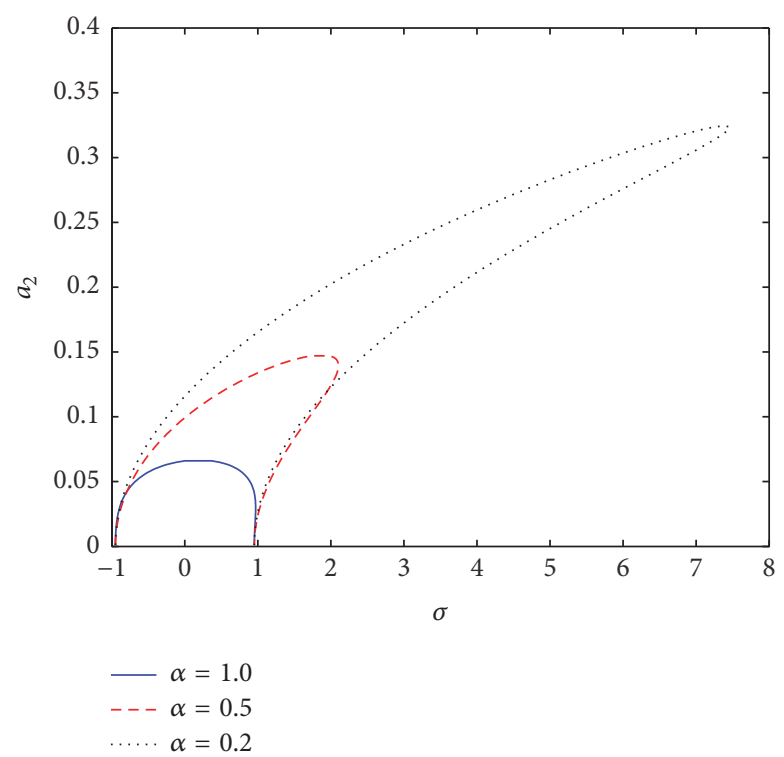

(b) The second-order mode

FIGURE 3: Influence of the fractional order on the steady-state responses based on Kirchhoff's model.

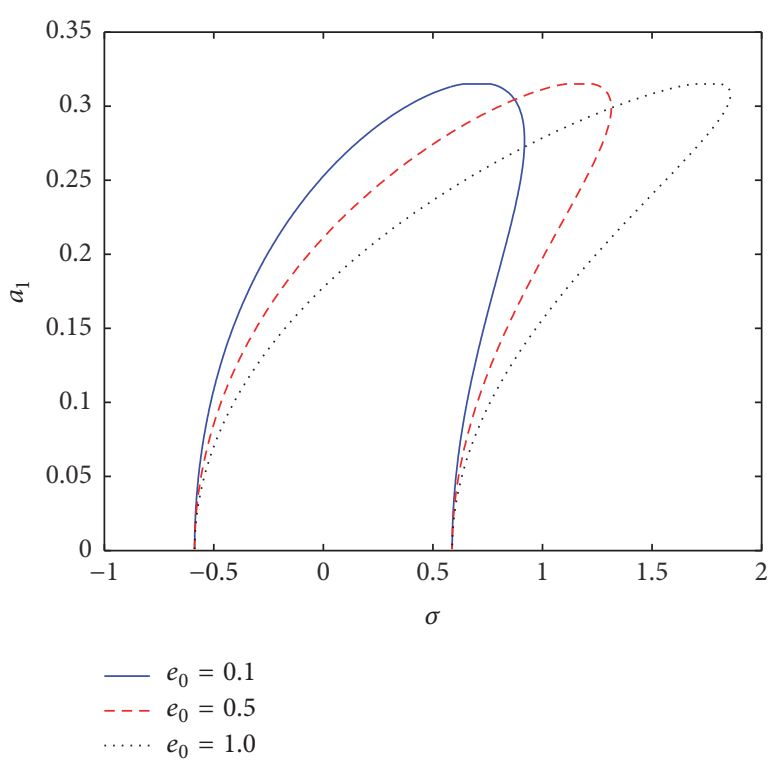

(a) The first-order mode

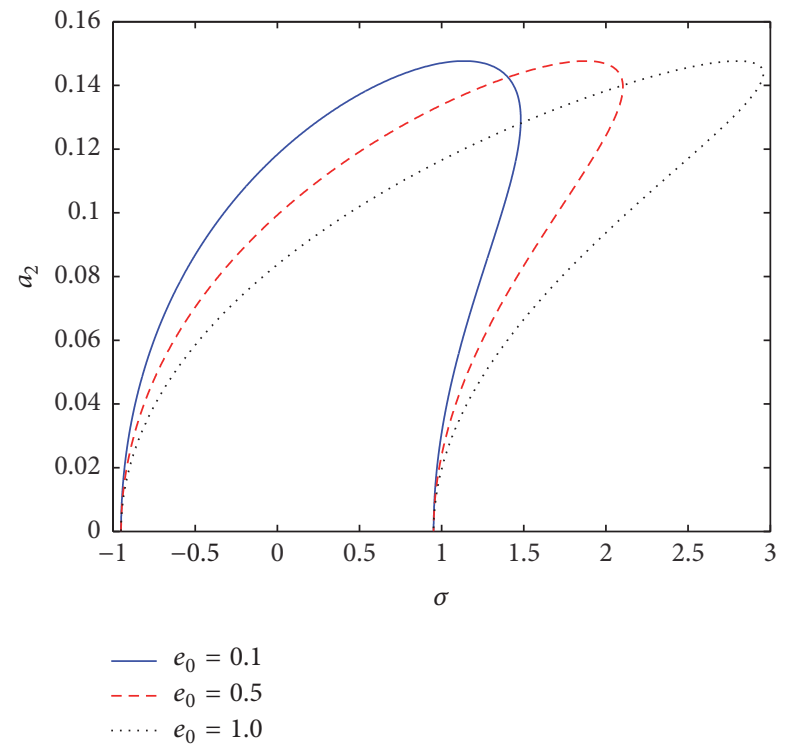

(b) The second-order mode

FIGURE 4: Influence of the stiffness constant on the steady-state responses based on Kirchhoff's fractional model.

model. Consider that the viscoelastic behavior of the string is described by the fractional Kelvin-Voigt constitutive relation; a new nonlinear fractional mathematical model governing transverse vibration of the string is derived from the Euler beam theory and Newton's second law. The direct multiscale method is applied to investigate the steady-state responses of the fractional damping string undergoing the principle parametric resonances. The investigation yields the following conclusions:
(1) The amplitudes and existing intervals of steady-state responses predicted by Kirchhoff's fractional model are much larger than those predicted by Mote's fractional model.

(2) In Kirchhoff's model, the fractional derivative has a significant effect on steady-state responses; the smaller fractional order can cause more rapid increasing in the response amplitudes and existing intervals. 


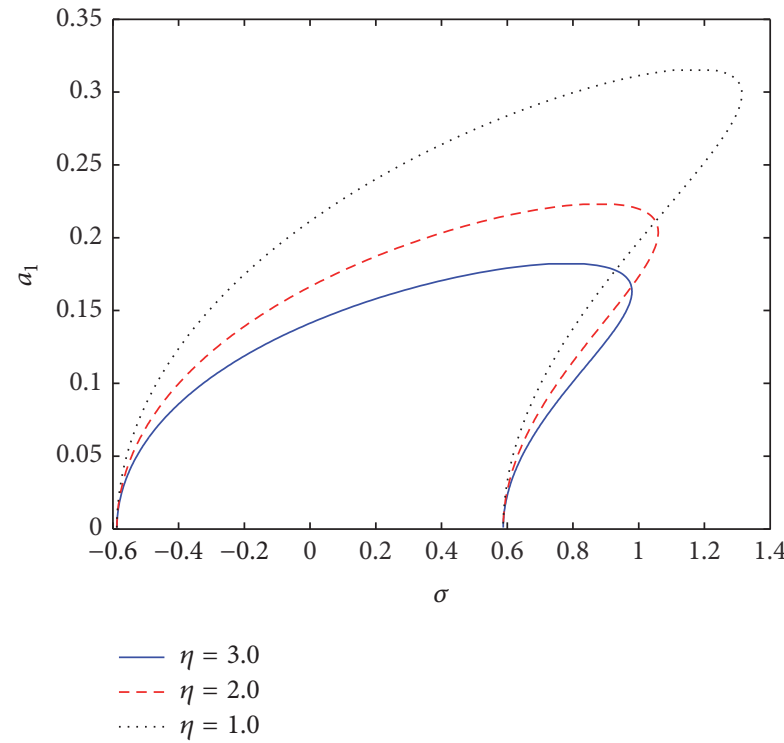

(a) The first-order mode

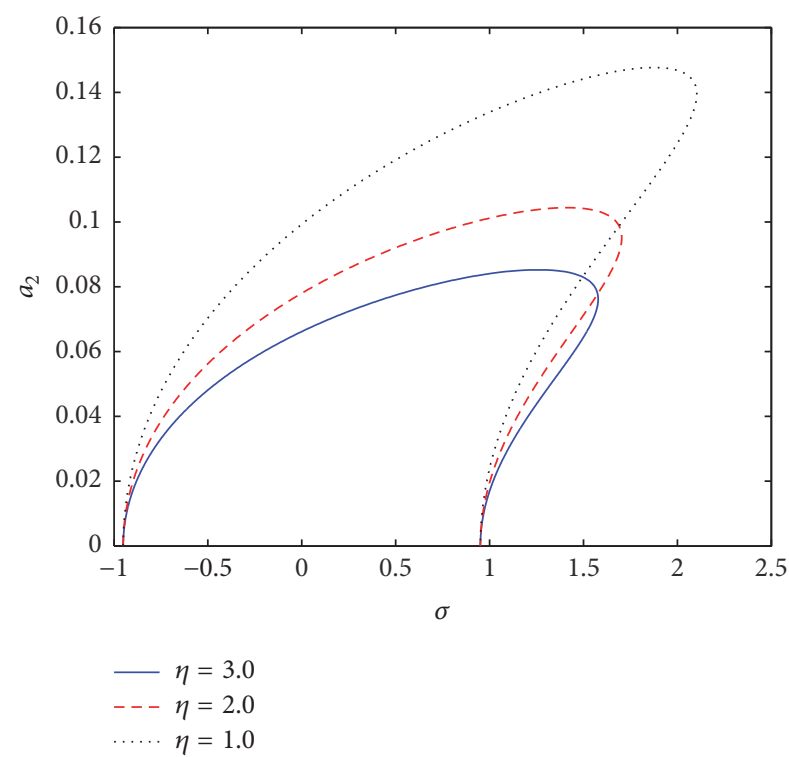

(b) The second-order mode

FIGURE 5: Effect of the viscosity coefficient on the steady-state responses based on Kirchhoff's fractional model.

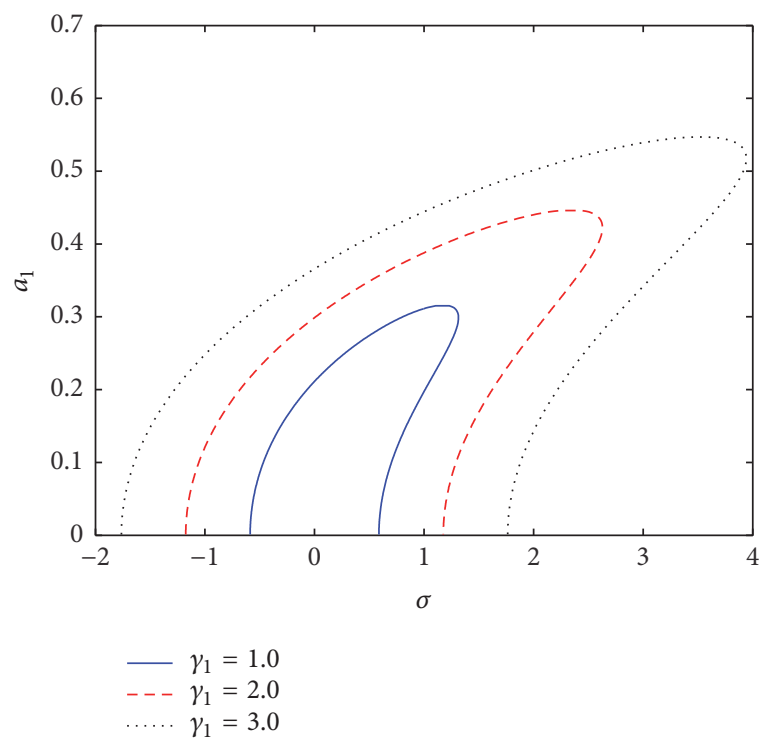

(a) The first-order mode

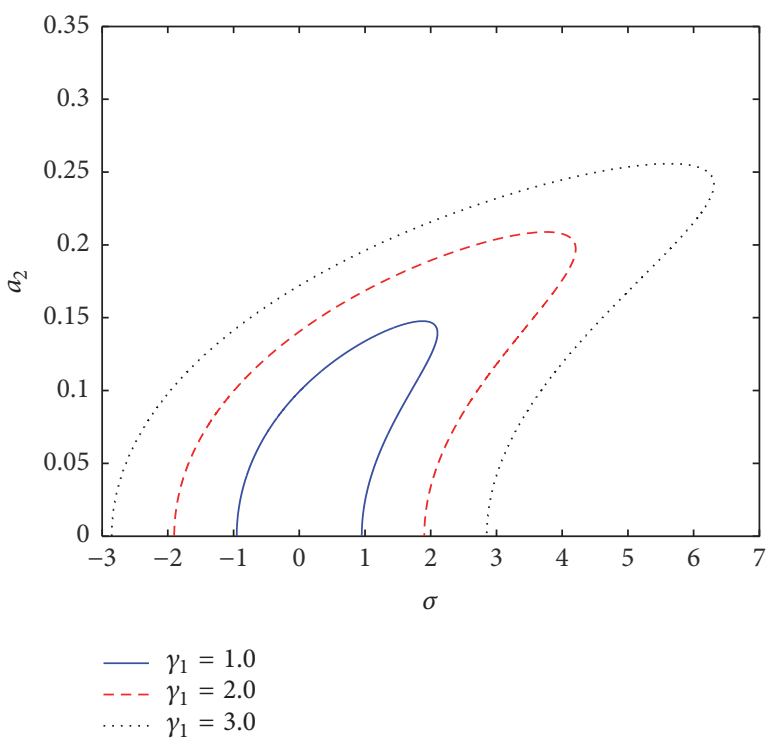

(b) The second-order mode

FIGURE 6: Effect of the axial-speed fluctuation amplitude on the steady-state responses based on Kirchhoff's fractional model.

(3) In Kirchhoff's fractional model, the response amplitudes decrease with the increasing of the stiffness constant and viscosity coefficient, while they increase as the axial-speed fluctuation amplitude increases. In addition, the larger the stiffness constant, the higher the axial-speed fluctuation amplitude; and the smaller viscosity coefficient tends to enlarge existing intervals of steady-state responses.

\section{Conflicts of Interest}

The authors declare that there are no conflicts of interest regarding the publication of this paper.

\section{Acknowledgments}

This work is supported by the Natural Science Research Project of Institutions of Higher Education in Anhui Province 
of China (nos. KJ2017A114, KJ2017A106, and TSKJ2016B18), the Promoting Program Project of Higher Education in Anhui province of China (no. TSSK2017B05), Research Fund of Young Teachers in Anhui Polytechnic University (no. 2016YQ06), the National Natural Science Foundation of China (nos. 11402087 and 51575001), and the Opening Fund of Key Laboratory of Mechanics, Anhui Polytechnic University (no. 201607).

\section{References}

[1] J. A. Wickert and C. D. Mote Jr., "Classical vibration analysis of axially moving continua," Journal of Applied Mechanics, vol. 57, no. 3, pp. 738-744, 1990.

[2] M. Pakdemirli, A. G. Ulsoy, and A. Ceranoglu, "Transverse vibration of an axially accelerating string," Journal of Sound and Vibration, vol. 169, no. 2, pp. 179-196, 1994.

[3] H. R. Öz, "Non-linear vibrations and stability analysis of tensioned pipes conveying fluid with variable velocity," International Journal of Non-Linear Mechanics, vol. 36, no. 7, pp. 10311039, 2001.

[4] L. H. Chen, W. Zhang, and Y. Q. Liu, "Modeling of nonlinear oscillations for viscoelastic moving belt using generalized Hamilton's principle," Journal of Vibration and Acoustics, vol. 129, no. 1, pp. 128-132, 2007.

[5] A. Yurddas, E. Özkaya, and H. Boyaci, "Nonlinear vibrations of axially moving multi-supported strings having non-ideal support conditions," Nonlinear Dynamics, vol. 73, no. 3, pp. 1223-1244, 2013.

[6] M. H. Ghayesh, M. Amabili, and M. P. Païdoussis, "Nonlinear dynamics of axially moving plates," Journal of Sound and Vibration, vol. 332, no. 2, pp. 391-406, 2013.

[7] T. G. Ritto, C. Soize, F. A. Rochinha, and R. Sampaio, "Dynamic stability of a pipe conveying fluid with an uncertain computational model," Journal of Fluids and Structures, vol. 49, pp. 412426, 2014

[8] G. Yao and Y. Zhang, "Reliability and sensitivity analysis of an axially moving beam," Meccanica, vol. 51, no. 3, pp. 491-499, 2016.

[9] X. D. Yang, M. Liu, Y. J. Qian, S. Yang, and W. Zhang, "Linear and nonlinear modal analysis of the axially moving continua based on the invariant manifold method," Acta Mechanica, vol. 228, no. 2, pp. 465-474, 2017.

[10] W. Wu, G. Jiang, S. Huang, and C. J. Leo, "Vertical Dynamic Response of Pile Embedded in Layered Transversely Isotropic Soil," Mathematical Problems in Engineering, vol. 2014, Article ID 126916, 2014.

[11] J. L. Sun, Y. Peng, H. M. Liu, and G. B. Jiang, "Vibration of moving strip with distribution stress in rolling process," Journal of Iron and Steel Research, International, vol. 17, no. 4, pp. 24-30, 2010.

[12] L. Ma, J. Chen, W. Tang, and Z. Yin, "Vibration-based estimation of tension for an axially travelling web in roll-to-roll manufacturing," Measurement Science and Technology, 2017.

[13] R. F. Fung, J. S. Huang, and Y. C. Chen, "The transient amplitude of the viscoelastic travelling string: An integral constitutive law," Journal of Sound and Vibration, vol. 201, no. 2, pp. 153-167, 1997.

[14] F. Pellicano, A. Fregolent, A. Bertuzzi, and F. Vestroni, "Primary and parametric non-linear resonances of a power transmission belt: Experimental and theoretical analysis," Journal of Sound and Vibration, vol. 244, no. 4, pp. 669-684, 2001.
[15] L. Q. Chen, X. D. Yang, and C. J. Cheng, "Dynamic stability of an axially accelerating viscoelastic beam," European Journal of Mechanics - A/Solids, vol. 23, no. 4, pp. 659-666, 2004.

[16] M. Pakdemirli and H. R. Öz, "Infinite mode analysis and truncation to resonant modes of axially accelerated beam vibrations," Journal of Sound and Vibration, vol. 311, no. 3-5, pp. 1052-1074, 2008.

[17] M. H. Ghayesh, "Stability characteristics of an axially accelerating string supported by an elastic foundation," Mechanism and Machine Theory, vol. 44, no. 10, pp. 1964-1979, 2009.

[18] S. M. Shahruz, "Stability of a nonlinear axially moving string with the kelvin-voigt damping," Journal of Vibration and Acoustics, vol. 131, no. 1, pp. 0145011-0145014, 2009.

[19] M. H. Ghayesh, "Nonlinear transversal vibration and stability of an axially moving viscoelastic string supported by a partial viscoelastic guide," Journal of Sound and Vibration, vol. 314, no. 3-5, pp. 757-774, 2008.

[20] T. Z. Yang, B. Fang, Y. Chen, and Y. X. Zhen, "Approximate solutions of axially moving viscoelastic beams subject to multifrequency excitations," International Journal of Non-Linear Mechanics, vol. 44, no. 2, pp. 230-238, 2009.

[21] B. Sahoo, L. N. Panda, and G. Pohit, "Two-frequency parametric excitation and internal resonance of a moving viscoelastic beam," Nonlinear Dynamics, vol. 82, no. 4, pp. 1721-1742, 2015.

[22] Z. Hou and J. W. Zu, "Non-linear free oscillations of moving viscoelastic belts," Mechanism and Machine Theory, vol. 37, no. 9, pp. 925-940, 2002.

[23] M. H. Yao, W. Zhang, and J. W. Zu, "Multi-pulse chaotic dynamics in non-planar motion of parametrically excited viscoelastic moving belt," Journal of Sound and Vibration, vol. 331, no. 11, pp. 2624-2653, 2012.

[24] S. Hatami, H. R. Ronagh, and M. Azhari, "Exact free vibration analysis of axially moving viscoelastic plates," Computers \& Structures, vol. 86, no. 17-18, pp. 1738-1746, 2008.

[25] Y. Q. Tang and L. Q. Chen, "Parametric and internal resonances of in-plane accelerating viscoelastic plates," Acta Mechanica, vol. 223, no. 2, pp. 415-431, 2012.

[26] K. Marynowski and T. Kapitaniak, "Zener internal damping in modelling of axially moving viscoelastic beam with time-dependent tension," International Journal of Non-Linear Mechanics, vol. 42, no. 1, pp. 118-131, 2007.

[27] L. Q. Chen, J. W. Zu, and J. Wu, "Steady-state response of the parametrically excited axially moving string constituted by the Boltzmann superposition principle," Acta Mechanica, vol. 162, no. 1-4, pp. 143-155, 2003.

[28] B. Wang and L. Q. Chen, "Asymptotic stability analysis with numerical confirmation of an axially accelerating beam constituted by the standard linear solid model," Journal of Sound and Vibration, vol. 328, no. 4-5, pp. 456-466, 2009.

[29] H. Ding and L. Q. Chen, "Stability of axially accelerating viscoelastic beams: multi-scale analysis with numerical confirmations," European Journal of Mechanics - A/Solids, vol. 27, no. 6, pp. 1108-1120, 2008.

[30] L. Q. Chen, W. J. Zhao, and J. W. Zu, “Transient responses of an axially accelerating viscoelastic string constituted by a fractional differentiation law," Journal of Sound and Vibration, vol. 278, no. 4-5, pp. 861-871, 2004.

[31] T. Z. Yang and B. Fang, "Asymptotic analysis of an axially viscoelastic string constituted by a fractional differentiation law," International Journal of Non-Linear Mechanics, vol. 49, pp. 170-174, 2013. 
[32] T. Z. Yang, X. Yang, F. Chen, and B. Fang, "Nonlinear parametric resonance of a fractional damped axially moving string," Journal of Vibration and Acoustics, vol. 135, no. 6, Article ID 064507, 2013.

[33] J. A. Wickert, "Non-linear vibration of a traveling tensioned beam," International Journal of Non-Linear Mechanics, vol. 27, no. 3, pp. 503-517, 1992.

[34] J. Moon and J. A. Wickert, "Non-linear vibration of power transmission belts," Journal of Sound and Vibration, vol. 200, no. 4, pp. 419-431, 1997.

[35] L. Q. Chen, H. Chen, and C. W. Lim, "Asymptotic analysis of axially accelerating viscoelastic strings," International Journal of Engineering Science, vol. 46, no. 10, pp. 976-985, 2008.

[36] Y. A. Rossikhin and M. V. Shitikova, "On fallacies in the decision between the Caputo and Riemann-Liouville fractional derivatives for the analysis of the dynamic response of a nonlinear viscoelastic oscillator," Mechanics Research Communications, vol. 45, pp. 22-27, 2012.

[37] K. B. Oldham and J. Spanier, The Fractional Calculus, Academic Press, New York, NY, USA, 1974.

[38] T. Z. Yang and B. Fang, "Stability in parametric resonance of an axially moving beam constituted by fractional order material," Archive of Applied Mechanics, vol. 82, no. 12, pp. 1763-1770, 2012.

[39] L. Q. Chen and J. W. Zu, "Solvability condition in multiscale analysis of gyroscopic continua," Journal of Sound and Vibration, vol. 309, no. 1-2, pp. 338-342, 2008. 


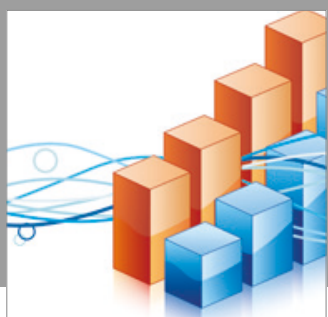

Advances in

Operations Research

vatersals

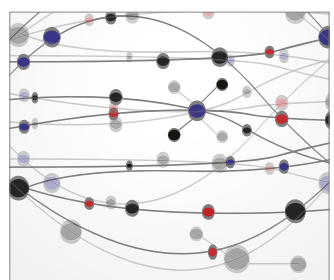

\section{The Scientific} World Journal
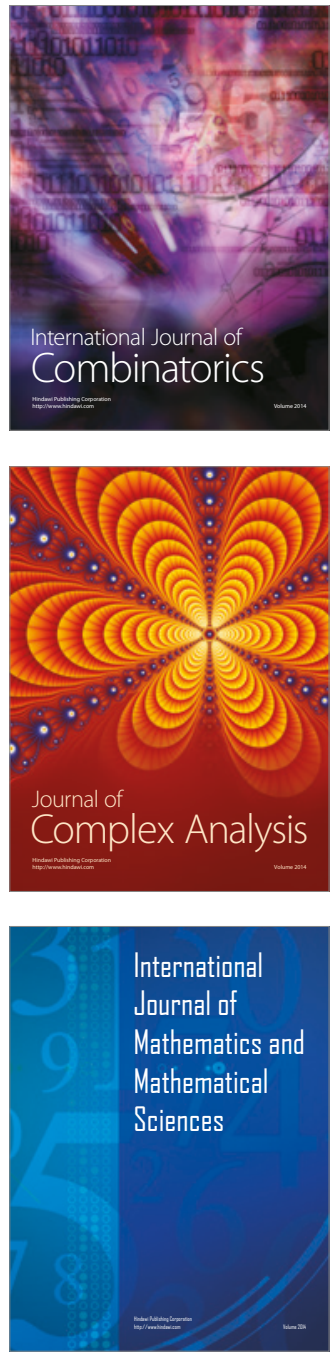
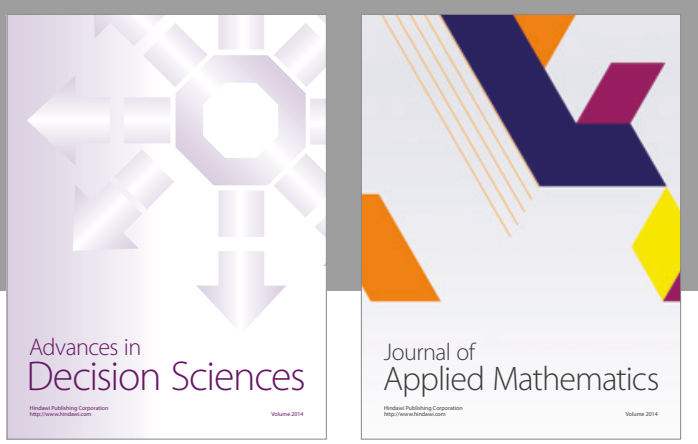

Algebra

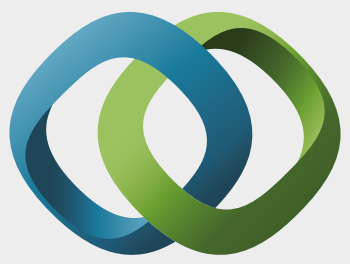

\section{Hindawi}

Submit your manuscripts at

https://www.hindawi.com
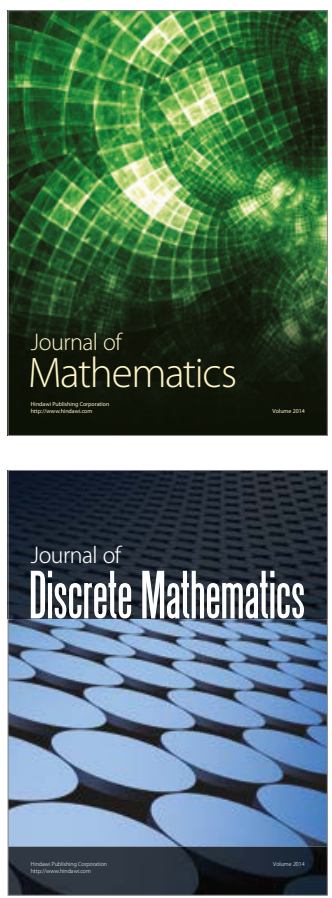

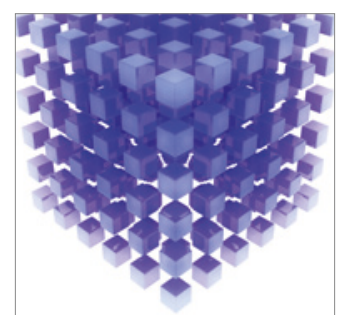

Mathematical Problems in Engineering
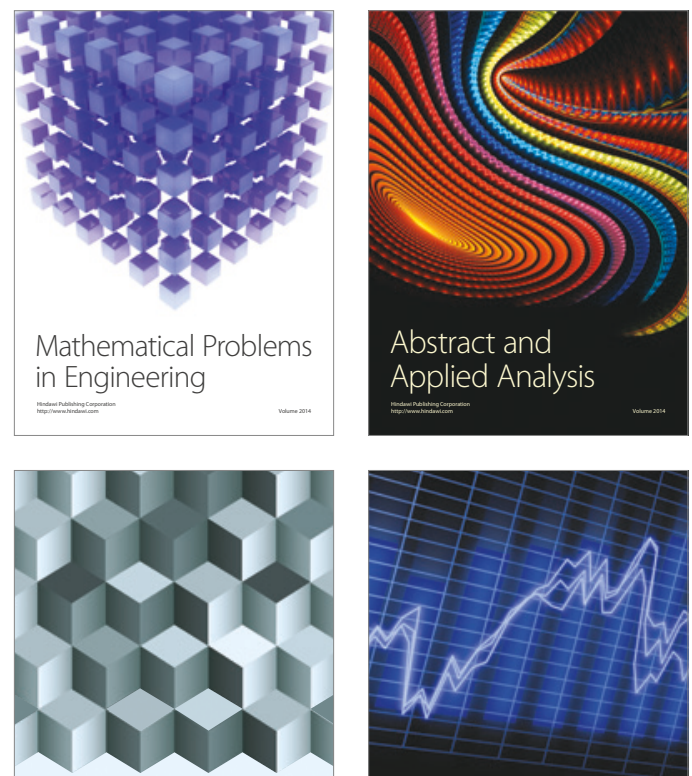

Journal of

Function Spaces

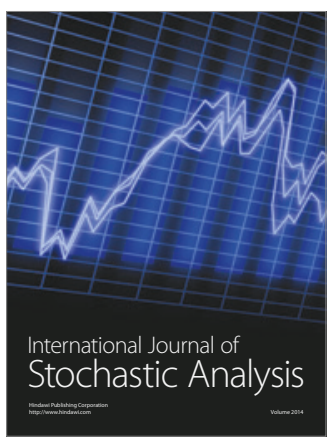

Probability and Statistics
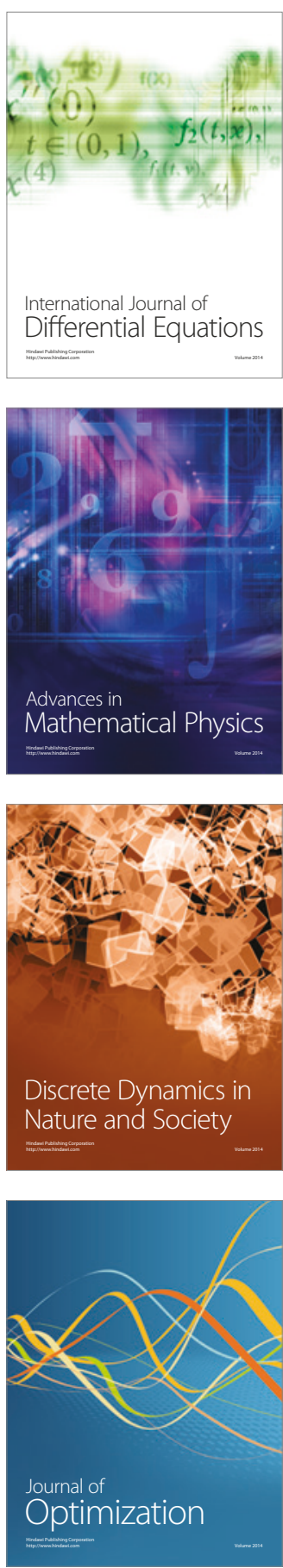